\title{
The longevity of posterior restorations in primary teeth
}

\author{
Abstracted from \\ Chisini LA, Collares K, Cademartori MG, de Oliveira LJC, Conde
}

MCM, Demarco FF, Corrêa MB. Restorations in primary teeth: a systematic review on survival and reasons for failures. Int J Paediatr Dent 2018; 28: 123-139.

Address for correspondence: Marcos Britto Corrêa, Graduate Program in Dentistry, Federal University of Pelotas, 457, Goncalves Chaves St. 5th floor, Pelotas 96015-560, Brazil. E-mail: marcosbrittocorrea@hotmail.com

\section{Question: What is the longevity of posterior restorations with different materials in primary teeth and what are the common reasons for failures?}

Data sources SciVerse Scopus, ISIS Web of Science, Cochrane library, Medline/PubMed. Studies published from 1996 to 2017 in English were considered.

Study selection Two independent reviewers screened the literature. Randomised clinical trials, non-randomised clinical trials with parallel groups or single group, retrospective studies) evaluating different posterior restorations (class I, class II restorations and crowns) with different materials (amalgam, compomer, composite, glass ionomer cement, stainless steel crown) placed in primary teeth by reporting different outcomes measures (survival rate, success rate, annual failure rate).

Data extraction and synthesis Two independent reviewers extracted data. Risk of bias was assessed using the Cochrane tool. A qualitative analysis was conducted.

Results Thirty-one studies were included. Seven different materials were used for restorations: amalgam (six studies), compomer (nine studies), composite (six studies), conventional glass ionomer cement (five studies), metal-reinforced glass ionomer cement (MRGIC) (four studies), resin-modified glass ionomer cement (ten studies), and stainless steel crown (SSC) (three studies). When considering the annual failure rate (AFR), composite showed the lowest (1.7-12.9\%) and MRGIC showed the highest (10.0-29.9\%). For the success rate, SSC presented the highest (96.1\%) and MRGIC presented the lowest (57.4\%). Class I restorations and restorations placed under the use of rubber dam revealed better results in both AFR and success rate. The main reason for failure was secondary caries.

Conclusions There is a large variation in longevity of posterior restorations in primary teeth. Secondary caries is the main reason causing failure.

\section{Commentary}

Dental caries remains a significant oral health problem in children all over the world. ${ }^{1}$ Dentists often use conventional restorative treatment to manage dental caries. Common restorative materials used include silver amalgam, composite, compomer, resinmodified glass ionomer cement, glass ionomer cement, composite strip crowns and stainless steel crowns. The longevity of dental restorations is not only related to the restorative material used, but also affected by clinical variables such as use of rubber dam, operators' ability and patients' characteristics. ${ }^{2}$ As there are no publications that summarised the longevity of restorations in primary teeth, the aim of this review was to investigate the failure rate and the main reasons of failures of posterior restorations in primary teeth.

The authors performed a literature search in four mainstream databases to identify studies which were published in English from 1996 to 2017. The rationale for restricting the time period of publications was not stated. The authors recruited all longitudinal clinical studies, including, but not limited to, randomised clinical trials. Hence, the majority of included studies (22/31) do not have a control group. None of the studies showed a low risk of bias in general, and most of the studies that were randomised revealed a high risk of bias in random sequence generation and allocation concealment. It is understandable that the blinding of operator (performance bias) cannot be achieved because of the nature of intervention. The authors did not conduct meta-analysis because of the high heterogeneity regarding study design and outcome measurement among the selected studies. However, they reported the overall failure rates for each restorative material by simply dividing a sum of the number of restorations evaluated by a sum of the number of failed restorations from different studies. This way of performing data analysis neglects the significant heterogeneity between studies, and thus the validity is in doubt. The authors presented the reasons for failure, which are recurrent caries, fracture of teeth, fracture or loss of restorations, endodontic complications etc. These reasons in fact are modes of failure, and the factors causing the failure were not reported in the review.

Aforementioned concerns would suggest that the quality of data extraction and analysis in this review is likely to be low. However, it is still a satisfying summary paper that fits with conventional wisdom around different restorative materials' longevity and provides valuable information around conventional restorative treatments in primary teeth, as long as the results are interpreted with some caution. The results of this review showed a large variation in longevity of posterior restoration with different materials in primary teeth. High quality randomised clinical trials on different dental restorative materials are in need to generate reliable results.

\section{Sherry Shiqian Gao}

Faculty of Dentistry, The University of Hong Kong, Hong Kong

1. Duangthip D, Chen KJ, Gao SS, Lo ECM, Chu CH. Managing Early Childhood Caries with Atraumatic Restorative Treatment and Topical Silver and Fluoride Agents. Int I Environ Res Public Health 2017; 14: pii:E1204.

2. Demarco FF, Corrêa MB, Cenci MS, Moraes RR, Opdam NJ. Longevity of posterior composite restorations: not only a matter of materials. Dent Mater 2012; 28: 87-101.

Evidence-Based Dentistry (2018) 19, 44. doi:10.1038/sj.ebd.6401302 